\title{
A psychological investigation of coronavirus disease 2019 (COVID-19) patients in mobile cabin hospitals in Wuhan
}

\author{
Haobin Zhang ${ }^{1 \#}$, Si Qin ${ }^{2,3 \#}$, Lei Zhang ${ }^{4}$, Zhuxiao Feng ${ }^{5}$, Changhe Fan ${ }^{5}$ \\ ${ }^{1}$ The Big Data Institute, Guangdong Create Environmental Technology Company Limited, Guangzhou, China; ${ }^{2}$ Department of Dermatology, \\ Guangdong Second Provincial General Hospital, Guangzhou, China; ${ }^{3}$ The Second School of Clinical Medicine, Southern Medical University, \\ Guangzhou, China; ${ }^{4}$ Department of Second General Surgery, Guangdong Second Provincial General Hospital, Guangzhou, China; ${ }^{5}$ Department of \\ Psychiatry, Guangdong Second Provincial General Hospital, Guangzhou, China \\ Contributions: (I) Conception and design: All authors; (II) Administrative support: None; (III) Provision of study materials or patients: None; (IV) \\ Collection and assembly of data: S Qin, L Zhang; (V) Data analysis and interpretation: H Zhang, S Qin; (VI) Manuscript writing: All authors; (VII) \\ Final approval of manuscript: All authors. \\ \#These authors contributed equally to this work. \\ Correspondence to: Changhe Fan. Department of Psychiatry, Guangdong Second Provincial General Hospital, Guangzhou, China. \\ Email: changhefan@yahoo.com.
}

\begin{abstract}
Background: This study investigated the depression, anxiety, and insomnia levels of coronavirus disease 2019 (COVID-19) patients admitted to two mobile cabin hospitals in Jianghan District (Wuhan, China).

Methods: Thirty COVID-19 (eight mild type and twenty-two common type) patients were evaluated using the Patient Health Questionnaire-9, the Generalized Anxiety Disorder 7 Questionnaire, the Insomnia Severity Index, and a semi-structured interview.

Results: All 30 patients reported varying degrees of anxiety, depression, and insomnia. The levels of depression and anxiety in mild type COVID-19 patients were significantly lower than those in common type COVID-19 patients. Significant improvements in depression $(\mathrm{P}<0.001)$ and anxiety $(\mathrm{P}<0.001)$ levels were found in the COVID-19 patients at the second evaluation compared with the baseline (admittance to hospital). More than $80 \%$ patients agreed that medical security, support from other patients, and a better living environment were the main reasons for improvements to their adverse psychological states.

Conclusions: Varying degrees of anxiety, depression, and insomnia frequently occur in patients with COVID-19. Standard treatment protocols and patient-centered care in the mobile cabin hospitals in this study provided the chance for COVID-19 patients to successfully improve their mental health during the outbreak of the pandemic.
\end{abstract}

Keywords: Coronavirus disease 2019 (COVID-19); patient psychology; mobile cabin hospital; depression; anxiety; insomnia; patient-centered care

Submitted Jun 01, 2020. Accepted for publication Jul 29, 2020.

doi: 10.21037/atm-20-4907

View this article at: http://dx.doi.org/10.21037/atm-20-4907

\section{Introduction}

Since December 2019, severe acute respiratory syndrome coronavirus 2 (SARS-CoV-2) has spread rapidly across the globe, affecting the physical and mental health of more than 2 million people worldwide (1). On January 31, 2020, the World Health Organization (WHO) listed coronavirus disease 2019 (COVID-19) as a public health emergency of international concern (PHEIC) (2). Because of the large number of infected people, the Chinese government has implemented substantial measures in the prevention and control of the epidemic and has reduced the spread of SARS-CoV-2. In Wuhan, the region of high incident of COVID-19 in China, more than 10 exhibition halls, gymnasiums, and other buildings were transformed into 
"mobile cabin hospitals" in various districts, to act as medical facilities for the early diagnosis and treatment of mild symptom patients and to relieve the burden of the public hospitals. During this public health emergency (3), COVID-19 patients have been found to be more likely to suffer from psychological problems, such as depression, anxiety and insomnia, than other people. Several psychological factors are thought to be related to this issue, such as fear of death, social distance from close family members and friends, and the inaccessibility of psychological management during the isolation period.

As clinicians working in Wuhan's mobile cabin hospitals (4), we prioritized patients' emotional comfort and psychological counseling, in order to help alleviate these psychological pressures. We not only provided patients with professional medical security and psychological care, but we also set up a reading corner in the hospital, where patients could access all kinds of books for free. Additionally, nurses regularly led patients in completing simple aerobic exercises. Patients were encouraged to communicate with one another during their admission periods at the mobile cabin hospital.

The present study presents the psychological questionnaires and semi-structured interview methods we used to collect, categorize, and analyze the depression, anxiety, insomnia, and related psychological conditions of COVID-19 patients treated in the mobile cabin hospitals. We hope our findings can provide a valuable reference for clinical psychological intervention work for use by medical professionals and hospital managers around the world. We present the following article in accordance with the SURGE reporting checklist (available at http://dx.doi.org/10.21037/ atm-20-4907).

\section{Methods}

\section{Design and data collection}

This was a study of COVID-19 patients receiving treatment in two mobile cabin hospitals, Jianghan Mobile Cabin Hospital and Jianghan Development Zone Mobile Cabin Hospital, in Wuhan, China, from February 5, 2020, to March 6, 2020. The patients were local residents from Jianghan District, Wuhan, and were arranged by the hospital to receive treatment in different areas under the charge of different medical teams through a completely random method. Inclusion criteria: (I) adult patients (ages ranging from 18 to 65 years old); (II) patients with a confirmed diagnosis of COVID-19 (mild type or common type) based on "The novel coronavirus pneumonia diagnosis and treatment plan (7th trial version)" in China (4) were invited to participate in the study through convenience sampling.

The diagnosis criteria (4) and clinical symptoms of the mild type and common type of COVID-19 were as follows:

- Patients in whom the nucleic acid of SARS-CoV-2 was found to be positive in the reverse transcription polymerase chain (RT-PCR) reaction test were considered COVID-19 patients;

* Patients with mild symptoms (fever and/or cough) and with no pneumonia evident from imaging examination were considered mild-type COVID-19;

* Patients with fever and respiratory tract symptoms, along with pneumonia evident from imaging examination, were considered common-type COVID-19;

* Patients affected by upper respiratory tract infections caused by other viruses were excluded.

Discharge criteria (4):

* Body temperature returns to normal for more than three days;

* Respiratory symptoms improved significantly;

* Pulmonary imaging showed significant improvement of acute exudative lesions;

* Two consecutive negative nucleic acid tests of sputum, nasopharyngeal swabs and other respiratory tract specimens. (Two sampling intervals at least 24 hours apart).

The study was conducted in accordance with the Declaration of Helsinki (as revised in 2013). The study was approved by Ethics Committee of Guangdong Second Provincial General Hospital (No.: 20200616-YXKXYJLW-01-01). All the candidates were informed of the study and consented to participate in the psychological questionnaires and interview survey without any incentives. Exclusion criteria: patients with schizophrenia, major depressive disorder, or other diagnosed mental health disorders, and/or those who were taking psychotropic drugs were excluded. The patients participated in the psychological questionnaires and face-to-face interviews separately, after being admitted to the hospital and after being informed that they could leave hospital. The same doctor was assigned to assist patients in completing the assessments and conducting the interviews. The psychological assessments and questionnaires were completed by the patients prior to the face-to-face interview with the doctor. The interviewer was able to check each 
Table 1 Details of assessments for depression, anxiety, and insomnia

\begin{tabular}{|c|c|c|c|}
\hline Variable & $\begin{array}{l}\text { Assessment } \\
\text { scale }\end{array}$ & Details & Scoring \\
\hline Depression & PHQ-9 & $\begin{array}{l}\text { Participants were asked to rate how often they } \\
\text { were bothered by the described symptoms (i.e., } \\
\text { the } 9 \text { DSM-IV criteria) over the past } 2 \text { weeks using } \\
\text { a } 4 \text {-point rating scale, ranging from } 0 \text { ("not at all") } \\
\text { to } 3 \text { ("every day") }\end{array}$ & $\begin{array}{l}\text { Total scores ranging from } 0 \text { to } 27 \text {, in which scores } \\
\text { of between } 0 \text { and } 4 \text { represent an absence of } \\
\text { depression, while scores of } 5-9,10-14,15-19 \text {, and } \\
20-27 \text { indicate mild, moderate, moderately severe, } \\
\text { and severe depression, respectively }\end{array}$ \\
\hline Insomnia & ISI & $\begin{array}{l}\text { Participants responded to items with reference to } \\
\text { a } 6 \text {-point rating scale, with scores ranging from } 0 \\
\text { to } 4 \text { (0= "not at all", } 1=\text { "mild", } 2=\text { "moderate", } 3= \\
\text { "severe", and } 4=\text { "extremely severe") }\end{array}$ & $\begin{array}{l}\text { Total scores ranging from } 0 \text { to } 28 \text {, in which a score } \\
\text { between } 0 \text { and } 7 \text { represents no clinically significant } \\
\text { insomnia, while scores of } 8-14,15-21 \text {, and } 22-28 \\
\text { indicate mild, moderately clinical, and severe } \\
\text { insomnia, respectively }\end{array}$ \\
\hline
\end{tabular}

DSM-IV, Diagnostic and Statistical Manual for Mental Disorders (4th ed.); GAD-7, Generalized Anxiety Disorder 7; ISI, Insomnia Severity Index; PHQ-9, Patient Health Questionaire-9.

recorded conversation to ensure the participants' utterances were accurately understood. Patients who did not complete all questionnaires and interviews will be classified as missing data. Response rate was calculated according to the percentage of patients who completed the investigation.

\section{Measures}

\section{Depression}

The Patient Health Questionnaire-9 (PHQ-9) $(5,6)$ was used to determine the presence of depression in the study's participants and assess its severity. The PHQ-9 is a scale consisting of nine items, based on the nine Diagnostic and Statistical Manual of Mental Disorder (DSM-IV) criteria (7), each of which is scored from 0 to 3 (Table 1). Using a cutoff point of $\geq 10$, the PHQ-9 has demonstrated a sensitivity of $88 \%$ and a specificity of $88 \%$ for the detection of major depression (6). Accordingly, it is considered to be a reliable, valid measure of depressive symptoms (Cronbach's $\alpha=0.85$ ) (5).

\section{Anxiety}

The Generalized Anxiety Disorder 7 (GAD-7) (8) scale was adopted to evaluate the presence of anxiety among our study's participants. This self-report measure assesses anxiety symptoms using seven-items that reflect the diagnostic criteria for generalized anxiety disorder (Table 1). It has shown good reliability (Cronbach's $\alpha=0.89$ ), and good criterion, construct, factorial, and procedural validity (9).

\section{Insomnia}

We adopted the Insomnia Severity Index (ISI) to assess sleep quality for patients. The ISI consists of seven items (see also Table 1), and was developed by Bastien et al. (10). It has been used widely to assess the severity of insomnia symptoms, and related reports indicate its high internal consistency (Cronbach's $\alpha=0.85$ ) (11).

In the process of evaluating to what degree the patients' depression, anxiety, and insomnia had improved, the following definitions and criteria were used: normal improvement was considered a change in symptoms from severe to moderate, moderate to mild, or mild to none; significant improvement was considered a change in symptoms from severe (or moderately severe) to mild (or none), or moderate to none.

After entering the mobile cabin hospital, patients in the study were asked the following two questions during a semistructured interview:

(I) "Were you worried during the isolation in hotels or schools in the past 2 weeks? If there were concerns, what was their main focus?"

(II) "Would you prefer to stay where you are or to enter the mobile cabin hospital for isolation where no extra drug treatment may not offered?" (When you enter the mobile cabin hospital for isolation, doctors may prescript the same medicine that you were taking in the hotel, school or extra medicine according to your condition).

A further two questions were asked to patients after they 
Table 2 Sociodemographic and clinical characteristics of the COVID-19 patients in the study

\begin{tabular}{|c|c|}
\hline Characters & Outcome \\
\hline \multicolumn{2}{|l|}{ Age (years) } \\
\hline$<30$ & 4 (13.33) \\
\hline $30-39$ & $9(30.0)$ \\
\hline $40-49$ & $6(20.0)$ \\
\hline $50-59$ & $9(30.0)$ \\
\hline$>60$ & $2(6.67)$ \\
\hline \multicolumn{2}{|l|}{ Occupation (medical-related work) } \\
\hline Yes & $1(3.33)$ \\
\hline No & $29(96.67)$ \\
\hline \multicolumn{2}{|l|}{ Sex ${ }^{b}$} \\
\hline Male & $15(50.0)$ \\
\hline Female & $15(50.0)$ \\
\hline \multicolumn{2}{|l|}{ Religion $^{b}$} \\
\hline Yes & 1 (3.33) \\
\hline No & $29(96.67)$ \\
\hline \multicolumn{2}{|l|}{ Location $^{\mathrm{b}}$} \\
\hline Local & $29(96.67)$ \\
\hline Non-local & $1(3.33)$ \\
\hline \multicolumn{2}{|l|}{ Marriage status $^{b}$} \\
\hline Married & $24(80.0)$ \\
\hline Unmarried & $6(20.0)$ \\
\hline \multicolumn{2}{|l|}{ COVID-19 type ${ }^{\mathrm{b}}$} \\
\hline Mild & $8(26.67)$ \\
\hline Common & 22 (73.33) \\
\hline \multicolumn{2}{|l|}{ Medical history ${ }^{\mathrm{b}}$} \\
\hline High blood pressure & $1(3.33)$ \\
\hline $\begin{array}{l}\text { Pregnancy-induced hypertension } \\
\text { syndrome }\end{array}$ & $1(3.33)$ \\
\hline Eczema & $1(3.33)$ \\
\hline None & 27 (90.0) \\
\hline
\end{tabular}

b, n (\%). COVID-19, coronavirus disease 2019.

met the discharge criteria:

(I) "Has the medical care in the hospital changed your mental state? How and why?"

(II) "Would you like to keep in contact with the doctors after leaving the hospital and to cooperate with them to complete a follow-up?”

\section{Data analysis}

The statistical analysis was conducted using IBM SPSS 24.0 software. The quantitative variables are expressed as mean \pm standard deviation (SD). Independent sample $t$-tests (or Wilcoxon rank-sum tests) were used to compare the relative levels of anxiety, depression, and insomnia of the COVID-19 patients after being admitted to mobile cabin hospital using the Shapiro-Wilk test of normality scores. Independent sample $t$-tests were used to compare the data when it passed the Shapiro-Wilk test. Wilcoxon rank-sum tests were used to compare the data when it didn't pass the Shapiro-Wilk test. Paired sample $t$-tests (or Wilcoxon matched-pairs signed-ranks tests ) were used to compare the relative levels of anxiety, depression, and insomnia of patients after entering the hospital and before leaving the hospital using the Shapiro-Wilk test of normality scores. Paired sample $t$-tests were used to compare the data when it passed the Shapiro-Wilk test. Wilcoxon matched-pairs signed-ranks tests were used to compare the data when it didn't pass the Shapiro-Wilk test. A P value $<0.05$ was considered to be statistically significant.

\section{Results}

\section{Sociodemographic characteristics}

The response rate was $100 \%$, and thirty patients with COVID-19 participated in the study. The mean (SD) age of patients was 42.5 (13.3) years old. The course of the disease ranged from 10 to 52 days, with the average course (SD) being 31 (12.6) days. Regarding medical history, 1 patient had a history of grade 2 hypertension, but the blood pressure was well controlled by conventional drugs; 1 patient had hypertension during pregnancy 4 years ago; and 1 patient had eczema. The remaining patients were generally healthy and had no underlying diseases or complications. During hospitalization, the blood pressure and glucose of all 30 patients remained at a normal level. In terms of occupation, only 1 patient was engaged in medical industry-related work. The sociodemographic and clinical characteristics of all participants are summarized in Table 2.

\section{Prevalence of depression, anxiety, and insomnia}

There was no significant difference found regarding 
Table 3 Comparison of anxiety, depression, and insomnia scores of participants following admission to the mobile cabin hospitals

\begin{tabular}{|c|c|c|c|c|c|c|c|}
\hline Variable & $\mathrm{n}$ & \multicolumn{2}{|c|}{ Anxiety } & \multicolumn{2}{|c|}{ Depression } & \multicolumn{2}{|c|}{ Insomnia } \\
\hline Sex & & & $\mathrm{t}=-0.267, \mathrm{P}=0.792$ & & $\mathrm{t}=0.708, \mathrm{P}=0.485$ & & $P=0.486$ \\
\hline Female & 15 & $10.27 \pm 1.580$ & & $11.20 \pm 2.077$ & & $8.73 \pm 2.052$ & \\
\hline Male & 15 & $10.40 \pm 1.121$ & & $10.73 \pm 1.486$ & & $8.13 \pm 1.552$ & \\
\hline Mild & 8 & $9 \pm 0.756$ & & $8.88 \pm 0.991$ & & $7.75 \pm 1.488$ & \\
\hline Common & 22 & $10.82 \pm 1.181$ & & $11.73 \pm 1.352$ & & $8.68 \pm 1.887$ & \\
\hline
\end{tabular}

$\begin{array}{lll}\text { Mild depression } & \text { No clinically significant insomnia } \\ \text { Moderate depression } & \text { Mild anxiety } & \text { Mild insomnia } \\ \text { Moderately severe depression } & \text { Moderate anxiety } & \end{array}$

Figure 1 COVID-19 patients with various degrees of anxiety, depression, and insomnia in the mobile cabin hospitals. COVID-19, coronavirus disease 2019.

depression $(\mathrm{t}=-0.267, \mathrm{P}=0.485)$, anxiety $(\mathrm{t}=0.708, \mathrm{P}=0.792)$, and insomnia (Wilcoxon rank-sum tests, $\mathrm{P}=0.486$ ) between the female patients and the male patients. However, the scores from the questionnaires differed according to the type of COVID-19 from which the patients were suffering. The levels of anxiety (Wilcoxon rank-sum tests, $\mathrm{P}<0.001$ ) and depression (Wilcoxon rank-sum tests, $\mathrm{P}<0.001)$ in patients with the common type of COVID-19 were significantly higher than those in patients with the mild type; no significant difference was found, however, in the scores concerning insomnia between the two types of patients (Wilcoxon rank-sum tests, $\mathrm{P}=0.202$ ), as shown in Table 3.

Evaluations of depression in the 30 mobile cabin hospital patients showed that 1 patient had moderately severe depression, 22 patients had moderate depression, and 7 patients had mild depression. The anxiety assessment showed that the study's participants had different degrees of anxiety: 23 of them had moderate anxiety, and 7 had mild anxiety. Among the investigated patients, only 8 did not suffer from insomnia, while the other 22 had mild insomnia. Figure 1 shows the varying degrees of anxiety, depression, and insomnia among the study's participants.

\section{Factors affecting COVID-19 patients' psychological states}

All 30 patients who participated in the questionnaire also took part in a semi-structured interview, after being admitted to the hospital, in order to explore which factors were affecting their psychological state; the results were as follows:

* Uncertain isolation time (100\%);

* Difficulty in adapting to the isolated environment $(93.3 \%)$;

* Ignorance of the physical condition (86.7\%);

* Fear of prognosis (56.7\%);

* Family reasons (16.7\%);

* Influence of disease on work (13.3\%).

Approximately $93.3 \%$ of patients $(n=28)$ were willing to enter the mobile cabin hospital for isolation treatment, even 
Table 4 Comparison of the scores from two evaluations of the study's COVID-19 patients

\begin{tabular}{lccc}
\hline Variable & First evaluation & Second evaluation & t-statistic, P value \\
\hline Anxiety scores $^{\mathrm{a}}$ & $10.33 \pm 1.348$ & $3.17 \pm 1.533$ & $\mathrm{P}<0.001$ \\
Depression scores $^{\mathrm{a}}$ & $10.97 \pm 1.790$ & $3.53 \pm 0.860$ & $\mathrm{P}<0.001$ \\
Insomnia scores $^{\mathrm{a}}$ & $8.43 \pm 1.813$ & $7.63 \pm 1.129$ & $\mathrm{t}=2.202, \mathrm{P}=0.036$ \\
\hline
\end{tabular}

${ }^{\mathrm{a}}$, mean \pm SD. COVID-19, coronavirus disease 2019 .

Table 5 Improvement of depression, anxiety, and insomnia symptoms in the study's COVID-19 patients

\begin{tabular}{lcccc}
\hline Variable & Patients $(\mathrm{n})$ & Significant improvement $(\%)$ & Improvement $(\%)$ & Non-improvement $(\%)$ \\
\hline Anxiety & 30 & $63.3(\mathrm{n}=19)$ & $36.7(\mathrm{n}=11)$ & $0(\mathrm{n}=0)$ \\
Depression & 30 & $63.3(\mathrm{n}=19)$ & $36.7(\mathrm{n}=11)$ & $0(\mathrm{n}=0)$ \\
Insomnia & 22 & $0(\mathrm{n}=0)$ & $50(\mathrm{n}=11)$ & $50(\mathrm{n}=11)$ \\
\hline
\end{tabular}

COVID-19, coronavirus disease 2019.

if without being given extra medication.

\section{Changes to COVID-19 patients' psychological states following treatment in hospital}

After entering the mobile cabin hospital for treatment, the 30 patients with COVID-19 who participated in the survey received a psychological assessment before leaving the hospital. The assessment results showed that there was some degree of change in their relative levels of anxiety, depression, and insomnia: 1 patient with moderately severe depression now had mild depression; 4 patients with moderate depression now had mild depression; 18 patients had recovered such that they were experiencing no depression; and 7 patients with mild depression completely recovered to no depression. Among the patients who had previously reported experiencing moderate anxiety, 4 were now experiencing only mild anxiety; 19 had reverted to a non-anxiety state; and 7 had completely returned to a nonanxiety state. Regarding insomnia, 11 patients had changed from mild insomnia to no insomnia, 11 reported no change in their insomnia levels, and 4 patients had changed from no insomnia to mild insomnia.

The levels of anxiety (Wilcoxon matched-pairs signedranks test, $\mathrm{P}<0.001$ ) and depression (Wilcoxon matched-pairs signed-ranks test, $\mathrm{P}<0.001)$ in the study's COVID-19 patients were significantly lower than those assessed at baseline; overall, the insomnia levels had significantly improved as compared to the levels reported by the patients on entry to the mobile hospital cabins, and the difference was statistically significant $(\mathrm{t}=2.202, \mathrm{P}=0.036)$ (Tables 4 and 5$)$.

\section{Factors in improving COVID-19 patients' psychological states}

All the COVID-19 who participated in the study consented to take part in semi-structured interviews before leaving the hospital, the purpose of which was to determine which factors contributed to the improvement of their respective psychological problems. The responses from the participants can be summarized as follows (Figure 2):

* Medical security (100\%);

* Support from other patients (93.3\%);

* Clean and orderly hospital environment (86.7\%);

* Sufficient life supplies (53.3\%);

- Cultural and recreational activities (reading and aerobics) (36.7\%).

Around $33.3 \%$ of the patients $(n=10)$ expressed their intention to keep in touch with their doctor after leaving the cabin hospital and to cooperate with hospital staff to complete psychological evaluation and follow-up. No correlation was found between the course of the disease and anxiety, depression, and insomnia levels.

\section{Discussion}

Since the outbreak of COVID-19, the socioeconomic wellbeing and quality of life of people worldwide have been dramatically affected $(1,2)$. As a catastrophic infectious disease, COVID-19's impact on patients' mental health 


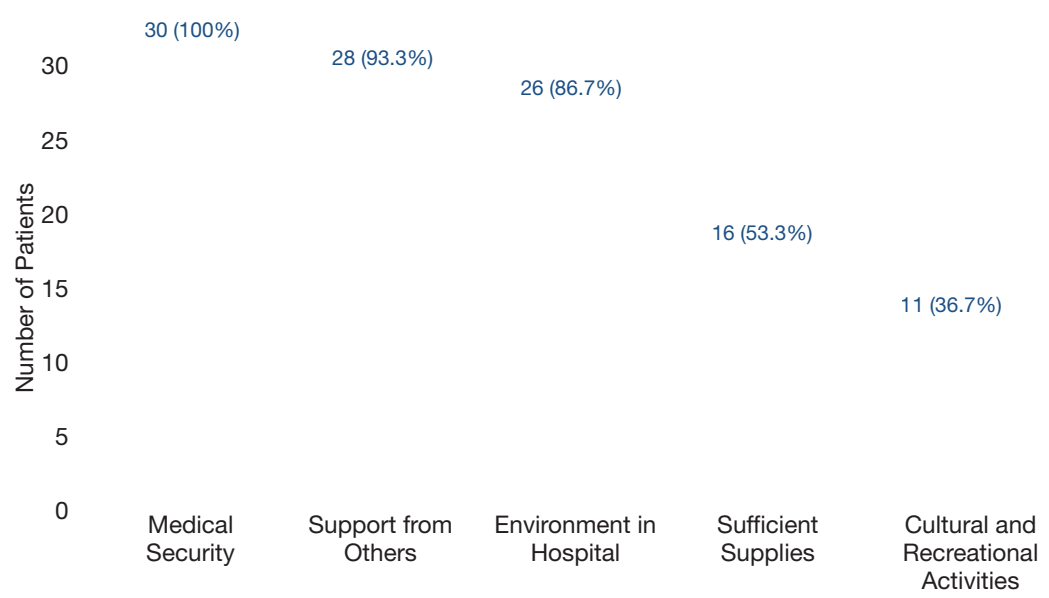

Figure 2 Causes of psychological changes of patients after treatment in the mobile cabin hospitals.

and psychological state can be complex (5). In Wuhan, the epicenter of COVID-19, a large number of patients were isolated in schools, hotels, and other areas so that appropriate, high-quality medical support could be provided for severe cases. On February 3, some buildings in Wuhan began to be transformed into mobile cabin hospitals (5), and medical personnel from all over China participated in the rescue efforts, aiming to provide medical security for patients with mild-type and common-type COVID-19. The present study focused on these types of patients who were admitted to the Jianghan Mobile Cabin Hospital and the Jianghan Development Zone Mobile Cabin Hospital. The study's 30 participating patients received standardized treatment, examination, and evaluation in these two mobile cabin hospitals, and were discharged after meeting the discharge criteria. We told the patient that after they entered the cabin hospital, the doctor would adjust the medication according to their physical condition, but when the original medication they had taken was appropriate, the doctor would not change the original medication regimen. Approximately 93.3\% of patients were willing to enter the mobile cabin hospital for isolation treatment, even if without being given extra medication. Most of them believed that they would get better medical care if they enter the mobile cabin hospital.

Recently, in order to improve the patient quality of life and satisfaction with treatment, a great deal of research on medical care models has been conducted across the globe. These studies have found that the adoption of a patient-centered approach is directly beneficial to patients, and is likely to achieve better treatment outcomes. This method places experts, clinicians, and patients on an equal position, and thus allows the healthcare providers to reach a better understanding of a patient's expectations, feelings, and social context regarding their illness (12), with these concerns being considered, discussed, and incorporated into treatment. Aspects of the patient-centered approach were integrated into our work in the mobile cabin hospitals in order to adapt the medical care to the individualized needs of each patient. In the present study, we found that the evaluation results pertaining to anxiety and depression in all patients had improved. More than half of the patients also reported improvement to their sleep patterns. The following sections share further details of our experiences and outcomes within the mobile cabin hospitals.

\section{Oral communication and interaction}

Clinicians were made available for face-to-face dialogue and interaction $(13,14)$ which were arranged at the patient's convenience. Conversations and consultations were conducted in private areas within the facility in order to establish a good rapport with patients. This dialogue would begin with the doctor's professional analysis of the patient's physical condition, followed by an explanation of any medication and the prognosis. Second, it was acknowledged that each patient was unique in terms of social resources, interpersonal relationships, advantages, limitations (14-16), and psychological states. It is imperative for a doctor to understand a patient's personal situation through talking to them, so as to be able to comfort the patient and relieve their tension and anxiety more effectively. Additionally, patients may acquire information about COVID-19 through their own channels, such as the Internet and books. Doctors can help patients determine 
the authenticity of this information and understand the disease more accurately through direct dialogue. Moreover, these exchanges of information are beneficial for clinicians, as they can be more informed of patients' individual expectations (17-19), which in turn increases patients' trust in doctors and promotes patient-healthcare provider concordance (20). Through our study's semistructured interviews, we found that more than $86.7 \%$ of patients reported their psychological status to be adversely affected by the uncertainty of their condition (e.g., lack of knowledge concerning the duration of isolation, their physical state, etc.), which could be better managed through dialogue and interaction.

\section{Improved health outcomes and medical security}

During their stay in the mobile cabin hospital, most of the patients experienced an improvement in their physical condition (21), and their confidence was further strengthened when they saw patients around them being discharged one after another. A small number of patients may suffer from disease fluctuation or adverse drug reactions, but the professional response and treatment from clinicians can help stabilize any negative emotions in these patients. In our interviews with the study's patients, we found that $100 \%$ of them believed that medical security was the main factor to improving their mental status. The security and support provided by our medical team in the mobile cabin hospitals included the diagnosis, treatment, and interpretation of patients' disease changes and adverse drug reactions by doctors as well as the medical care provided by nurses. In addition, regular police patrols in the ward provided patients with a sense of security.

\section{Sharing experiences and giving encouragement}

Conversations among patients facilitated experience sharing $(14,22)$. Patients talked to one another about the difficulties they encountered and how to deal with their own anxiety and depression. Doctors and patients also shared their experiences and gave each other encouragement concerning both the disease and life in general. It is also worth mentioning that there was a wall in both of the mobile cabin hospitals on which paintings from the children of medical team members were placed. These had the theme of encouraging patients to "get well soon". These walls also featured notes and messages for and from patients encouraging themselves and others. As gifts, some of the paintings were sent to particular patients, most of whom affixed the paintings to their beds. It should be acknowledged as well that the outbreak has generated a great deal of negative emotions in patients, likely stemming from a loss of income, stigma, and side effects (23). In the cabin hospitals, we perceived there to be a harmonious environment in which patients were not subject to the pressure or scrutiny of the wider society. More than $93 \%$ of patients in our study reported benefiting from the encouragement and exchange of experiences with other patients; thus experience sharing may be a good way to begin the release of negative emotions.

\section{Avoiding frequent job rotation}

Some clinicians believe that avoiding frequent job rotation may enhance patients' sense of security $(14,24)$ and increase their trust in healthcare professionals. Accordingly, in the mobile cabin hospitals, doctors tracked the physical condition and psychological states of 30 to 50 patients, so that patients do not need to have discussions with multiple doctors. It is clearly easier for doctors to maintain a harmonious environment once they are familiar with a patient's personality, condition, and background. Moreover, doctors have different personality types that can offer different levels of support. For example, easygoing and optimistic doctors are more likely to offer psychological support. Doctors with different approaches may have variable effects on patients' psychologies. All of the doctors working in the mobile cabin hospitals underwent psychological training which emphasized the need for empathy and good communication skills.

\section{Rehabilitation program}

An important aspect of patient-centered care is accepting the patient as an active part of his or her healthcare plan and as an agentive member of society $(14,17,25)$. In the mobile cabin hospital, the staff set up a Wi-Fi network in order to provide patients with access to the Internet. Entertainment facilities, including a reading corner and fitness equipment such as jumping ropes and elastic belts, were provided for patients to enjoy in their spare time. Some activities such as taiji (tai chi) and square dancing were also provided by the nurses to facilitate patients' integration into the new environment $(14,26)$. These measures can effectively ensure that patients avoid paying excessive attention to their disease and instead focus on improving their quality of life. 
As patients choose rehabilitation activities according to their own condition, they also have the opportunity to address and satisfy their unique needs, such as those of exercise, recreation, and nutrition, under the guidance of a doctor.

To summarize, it was found that the centralized treatment approach of the mobile hospitals offers an effective way for the psychological intervention team to identify individuals who require psychological treatment, which can ensure the physical and mental health of patients overall (27-29).

\section{Strength and limitations}

One important strength of this study is that it was situated within the high-density population of Wuhan, with a significant COVID-19 disease burden and corresponding mental disorders. In addition, the mobile cabin hospitals in Wuhan handled a large number of patients in a short time. Therefore, all COVID-19 patients suffer from similar social and environmental impacts, which can reduce confounders compared to psychological studies conducted over a longer period of time or in different regions. Furthermore, the study explored the levels of and changes in depression, anxiety, and insomnia symptoms in COVID-19 patients, and clarifying the related factors of these conditions is more conducive to targeted psychological intervention and improving the efficiency of targeted treatments (30). However, the small sample size, for the purposes of meaningful comparison, may be considered a limitation of the present study. We were unable to access all the patients who were in the mobile hospitals, as some of them were managed by other medical teams, and their inclusion may have yielded additional insights. Nonetheless, with COVID-19 continuing to spread, our finding that the successful improvement of a patient's mental health hinges on patient-centered care should assist in the future and ongoing treatment of affected individuals.

\section{Conclusions}

A high proportion of COVID-19 patients in the mobile cabin hospitals experienced varying degrees of depression, anxiety, and insomnia. Following standard treatment and patient-centered care in the hospital, both the anxiety and depression levels of all the patients were found to have improved to some extent. This suggests that the mobile cabin hospital setting not only provided a strong guarantee of medical security for a large number of COVID-19 patients during the peak of the pandemic, but also provided a significant amount of psychological comfort for patients when confronting this public health emergency.

\section{Acknowledgments}

We would like to thank Dr. Kaiyi Wang for helping in polishing the language and revising the final paper.

Funding: None.

\section{Footnote}

Reporting Checklist: The authors have completed the SURGE reporting checklist. Available at http://dx.doi. org/10.21037/atm-20-4907

Data Sharing Statement: Available at http://dx.doi. org/10.21037/atm-20-4907

Conflicts of Interest: All authors have completed the ICMJE uniform disclosure form (available at http://dx.doi. org/10.21037/atm-20-4907). The authors have no conflicts of interest to declare.

Ethical Statement: The authors are accountable for all aspects of the work in ensuring that questions related to the accuracy or integrity of any part of the work are appropriately investigated and resolved. The study was conducted in accordance with the Declaration of Helsinki (as revised in 2013). The study was approved by Ethics Committee of Guangdong Second Provincial General Hospital (No.: 20200616-YXKXYJ-LW-01-01). All the candidates were informed of the study and consented to participate in the psychological questionnaires and interview survey without any incentives.

Open Access Statement: This is an Open Access article distributed in accordance with the Creative Commons Attribution-NonCommercial-NoDerivs 4.0 International License (CC BY-NC-ND 4.0), which permits the noncommercial replication and distribution of the article with the strict proviso that no changes or edits are made and the original work is properly cited (including links to both the formal publication through the relevant DOI and the license). See: https://creativecommons.org/licenses/by-nc-nd/4.0/.

\section{References}

1. Coronavirus disease (COVID-19) Pandemic Available 
online: https://www.who.int/emergencies/diseases/novelcoronavirus-2019 (Accessed Mar 4, 2020).

2. WHO Timeline - COVID-19. Available online: https:// www.who.int/zh/news-room/detail/08-04-2020-whotimeline---covid-19 (Accessed Mar 4, 2020).

3. Zhu N, Zhang D, Wang W, et al. A novel coronavirus from patients with pneumonia in China, 2019. N Engl J Med 2020;382:727-33.

4. Yang L, Jiang L, Bai Y, et al. Efficient management procedures for suspected or mild novel coronavirus pneumonia cases at mobile cabin hospital. Journal of Third Military Medical University 2020;42:545-8.

5. Kroenke K, Spitzer RL, Williams JB. The PHQ-9:validity of a brief depression severity measure. J Gen Intern Med 2001;16:606-13.

6. Fata Nahas ARM, Elkalmi RM, Al-Shami AM, et al. Prevalence of depression among health sciences students: findings from a public university in Malaysia. J Pharm Bioallied Sci 2019;11:170-5.

7. The National Health Commission of the People's Republic of China. The novel coronavirus pneumonia diagnosis and treatment plan (trial, 7th ed.). Available online: http:// www.nhc.gov.cn/xcs/zhengcwj/202003/46c9294a7dfe4cef8 0dc7f5912eb1989.shtml (Accessed Mar 4, 2020).

8. Spitzer RL, Kroenke K, Williams JB, et al. A brief measure for assessing generalized anxiety disorder: the GAD-7. Arch Intern Med 2006;166:1092-7.

9. Löwe B, Decker O, Müller S, et al. Validation and standardization of the generalized anxiety disorder screener (GAD-7) in the general population. Med Care 2008;46:266-74.

10. Bastien CH, Vallières A, Morin CM. Validation of the Insomnia Severity Index as an outcome measure for insomnia research. Sleep Med 2001;2:297-307.

11. Chung KF, Kan KK, Yeung WF. Assessing insomnia in adolescents: comparison of Insomnia Severity Index, Athens Insomnia Scale, and Sleep Quality Index. Sleep Med 2011;12:463-70.

12. McWhinney IR. Patient-centred and doctor-centred models of clinical decision-making. In: Sheldon M, Brooke J, Rector A. editors. Decision-making in general practice. London: Palgrave, 1985:31-46.

13. Edvardsson D, Fetherstonhaugh D, Nay R. Promoting a continuation of self and normality: person-centred care as described by people with dementia, their family members and aged care staff. J Clin Nurs 2010;19:2611-8.

14. Røsvik J, Kirkevold M, Engedal K, et al. A model for using the VIPS framework for person-centred care for persons with dementia in nursing homes: a qualitative evaluative study. Int J Older People Nurs 2011;6:227-36.

15. Dudas K, Olsson LE, Wolf A, et al. Uncertainty in illness among patients with chronic heart failure is less in personcentred care than in usual care. Eur J Cardiovasc Nurs 2013;12:521-8.

16. Røen I, Kirkevold Ø, Testad I, et al. Person-centered care in Norwegian nursing homes and its relation to organizational factors and staff characteristics: a crosssectional survey. Int Psychogeriatr 2018;30:1279-90.

17. Coyne I, Holmström I, Söderbäck M. Centeredness in healthcare: a concept synthesis of family-centered care, person-centered care and child-centered care. J Pediatr Nurs 2018;42:45-56.

18. Geboy L. Linking person-centered care and the physical environment: 10 design principles for elder and dementia care staff. Alzheimer's Care Today 2009;10:228-31.

19. Rubashkin N, Warnock R, Diamond-Smith N. A systematic review of person-centered care interventions to improve quality of facility-based delivery. Reprod Health 2018;15:169.

20. Hung L, Chaudhury H, Rust T. The effect of dining room physical environmental renovations on person-centered care practice and residents' dining experiences in longterm care facilities. J Appl Gerontol 2016;35:1279-301.

21. Abbott KM, Heid AR, Van Haitsma K. "We can't provide season tickets to the opera": Staff perceptions of providing preference-based person-centered care. Clin Gerontol 2016;39:190-209.

22. Edvardsson D, Sandman PO, Borell L. Implementing national guidelines for person-centered care of people with dementia in residential aged care: effects on perceived person-centeredness, staff strain, and stress of conscience. Int Psychogeriatr 2014;26:1171-9.

23. Gebremariam MK, Bjune GA, Frich JC. Barriers and facilitators of adherence to TB treatment in patients on concomitant TB and HIV treatment: a qualitative study. BMC Public Health 2010;10:651.

24. Boscart VM, Davey M, Ploeg J, et al. Psychometric evaluation of the Team Member Perspectives of PersonCentered Care (TM-PCC) survey for long-term care homes. Healthcare (Basel) 2018;6:59.

25. Fawcett TJ, Rhynas SJ. Re-finding the 'human side' of human factors in nursing: helping student nurses to combine person-centred care with the rigours of patient safety. Nurse Educ Today 2014;34:1238-41.

26. Alharbi TS, Ekman I, Olsson LE, et al. Organizational culture and the implementation of person-centered care: 
results from a change process in Swedish hospital care. Health Policy 2012;108:294-301.

27. Hartung TJ, Friedrich M, Johansen C, et al. The Hospital Anxiety and Depression Scale (HADS) and the 9-item Patient Health Questionnaire (PHQ-9) as screening instruments for depression in patients with cancer. Cancer 2017;123:4236-43.

28. Driessen E, Abbass AA, Barber JP, et al. Which patients benefit specifically from short-term psychodynamic psychotherapy (STPP) for depression? Study protocol

Cite this article as: Zhang H, Qin S, Zhang L, Feng Z, Fan C. A psychological investigation of coronavirus disease 2019 (COVID-19) patients in mobile cabin hospitals in Wuhan. Ann Transl Med 2020;8(15):941. doi: 10.21037/atm-20-4907 of a systematic review and meta-analysis of individual participant data. BMJ Open 2018;8:e018900.

29. Duan L, Zhu G. Psychological interventions for people affected by the COVID-19 epidemic. Lancet Psychiatry 2020;7:300-2.

30. Noormohammadpour P, Fakour Y, Nazemei MJ, et al. Evaluation of some psychological factors in psoriatic patients. Iran J Psychiatry 2015;10:37-42.

(English Language Editor: J. Gray) 